Letrônica, Porto Alegre, v. 7, n. 2, p. 795-820, jul./dez., 2014

\title{
AQUISIÇÃO DA LATERAL SILÁBICA DO INGLÊS: UMA ANÁLISE VIA TEORIA DA OTIMALIDADE ESTOCÁSTICA
}

\author{
ENGLISH SYLLABIC-LATERAL ACQUISITION: \\ AN ANALYSIS VIA STOCHASTIC OPTIMALITY THEORY
}

\author{
Fernando Cabral Alves* \\ Rubens Marques de Lucena*
}

\begin{abstract}
Resumo: Este artigo busca principalmente propor um modelo de gramática que descreva e explique o processo de aquisição da lateral silábica do inglês por aprendizes brasileiros. Pretendemos responder às seguintes questões: 1) Qual gramática está sendo adquirida? Mais especificamente: qual é a gramática que licencia a forma [ł] na língua inglesa de falantes nativos (bem como suas variantes)? 2) Quais são as principais etapas pelas quais a gramática da interlíngua do aprendiz brasileiro no processo de aquisição de [ł] passa? 3) Como explicar a variação na interlíngua durante todo o processo de aquisição? É possível prever as variantes que irão emergir no processo de aquisição de [ł] juntamente com suas frequências e estabelecer os limites da variabilidade? Para responder tais questões, fazemos uso da Teoria da Otimalidade em sua versão Estocástica e seu Gradual Learning Algorithm (BOERSMA \& HAYES, 2001). Nosso modelo foi embasado e testado por dados empíricos de um estudo realizado por nós assim como de outros autores.
\end{abstract}

Palavras-chave: Variação linguística; Teoria da Otimalidade Estocástica; Aquisição de L2.

\begin{abstract}
This paper seeks mainly to propose a grammatical model capable of describing and explaining the acquisition process of the English syllabic lateral by Brazilian learners. We aim to answer the following questions: 1) What grammar is being acquired? More specifically: what grammar allows the form [ $[1]$ to emerge as an output in L1 English (as well as its variants)? 2) What are the main stages through which the interlanguage grammar of a Brazilian learner goes in trying to acquire [ł]? 3) How to explain variation in the interlanguage throughout the acquisition process? Is it possible to predict what forms will emerge during the acquisition process along with their frequencies and set the limits for variability? In order to answer these questions, we have made use of the Stochastic version of Optimality Theory and its Gradual Learning Algorithm (BOERSMA \& HAYES, 2001). Our model has been based on and tested by empirical data collected in a study of ours and those of others.
\end{abstract}

Keywords: Linguistic variation; Stochastic Optimality Theory; L2 Acquisition.

\footnotetext{
* Graduado em Letras, com licenciatura em língua inglesa, pela Universidade Federal da Paraíba. É professor bolsista do programa Inglês sem Fronteiras na UFPB.

*Doutor em Linguística pela Universidade Federal da Paraíba. Atualmente é Professor Adjunto da Universidade Federal da Paraíba, onde atua junto ao Departamento de Letras Estrangeiras Modernas (DLEM) e ao Programa de Pós-Graduação em Linguística (PROLING).
} 


\section{Introdução}

Nosso trabalho parte de dois problemas. Primeiramente, parece não haver uma descrição e explicação teórica satisfatória sobre os fenômenos relacionados à lateral silábica do inglês L1. Como veremos na seção dedicada à caracterização do objeto de estudo (2), não há, por exemplo, um consenso sobre qual seja a forma subjacente das sílabas onde a lateral silábica emerge como output (ODA, 2014). Em segundo lugar, nas poucas vezes em que a aquisição da lateral silábica por aprendizes brasileiros de inglês foi estudada, ela recebeu uma atenção secundária. Um maior destaque vem sendo dado à aquisição da lateral em posição de coda (HAHN, 2010).

Esses problemas são o reflexo de uma escassez de trabalhos preocupados com a formalização desse fenômeno tanto na gramática do falante adulto de inglês L1 quanto na das interlínguas dos aprendizes dessa língua. Na Teoria da Otimalidade (PRINCE \& SMOLENSKY, 1993) (doravante OT), por exemplo, não há, até onde conhecemos, qualquer trabalho que proponha formalizar a ocorrência da lateral silábica do inglês e testar suas predições contra sua real distribuição em dados empíricos. Uma rápida busca no portal online de pesquisas em OT (http://roa.rutgers.edu) nos revela tal ausência. Se fizermos uma busca pela palavra-chave 'syllabic' (nem precisamos ser tão específicos em relação à lateral), encontraremos apenas um trabalho que se relaciona de fato com as consoantes silábicas ${ }^{1}$, mas ainda assim não tratam da questão no inglês.

No intuito de suprir essa lacuna encontrada nos estudos de fonologia, este trabalho tem como principal objetivo propor um modelo do processo de aquisição da lateral silábica do inglês por aprendizes brasileiros e testá-lo com os dados empíricos coletados em um estudo nosso e no de outros autores. Para chegarmos a esse objetivo precisamos também formalizar a gramática da lateral silábica no inglês L1. 0 modelo fará uso da OT em sua versão Estocástica (doravante SOT) e de seu Algoritmo de Aprendizagem Gradual (Gradual Learning Algorithm) (BOERSMA \& HAYES, 2001). Acreditamos que, embora estejamos lidando com um fenômeno pouco estudado, a partir das ferramentas teóricas da SOT seja possível construir um modelo que descreva e

\footnotetext{
10 trabalho de Piñeros (2014) sobre a formação de consoantes silábicas no espanhol tradicional do Novo México.
} 
explique o processo de aquisição de [ł] de maneira a responder às seguintes questões norteadoras:

1) Qual gramática está sendo adquirida? Mais especificamente: qual é a gramática que licencia a forma $[\nmid]$ na língua inglesa de falantes nativos?

2) Quais são as etapas pelas quais a gramática da interlíngua do aprendiz brasileiro no processo de aquisição de [ł] passa?

3) Como explicar a variação na interlíngua durante todo o processo de aquisição? Em outras palavras, como prever as variantes que irão emergir no processo de aquisição de [ł] (inclusive os limites da opcionalidade), assim como a frequência dessas variantes?

Nossas respostas para essas perguntas virão a partir da análise de dados e do modelo proposto na seção 4 deste artigo. Contudo, antes precisamos entender melhor nosso objeto de estudo (seção 1) e a metodologia da coleta dos dados que irão ser utilizados como subsídio empírico para testar nosso modelo (seção 2).

\section{Caracterização do objeto de estudo}

\subsection{A lateral no inglês}

Em língua inglesa, a consoante lateral pode ocorrer no ataque, no núcleo, e na coda silábica. Sua ocorrência em posição de núcleo se dá em sílabas fracas, como nas últimas sílabas das palavras ['pi:pł] 'people', ['ketł̣] 'kettle', e ['næ[nłł] 'national. 0 fenômeno é quase categórico em língua inglesa, mas ainda apresenta um pequeno grau de variação com o schwa. Portanto, existe a possibilidade de as palavras acima serem

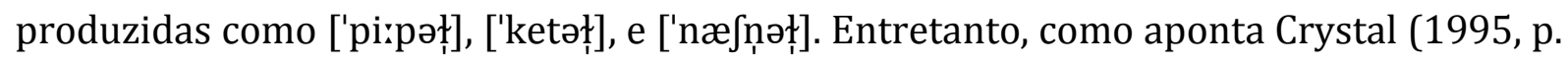
$246)^{2}$ "these pronunciations are highly artificial and would never be heard in usual conversational speech". Dados empíricos corroboram a afirmação do autor. Em um experimento realizado por ZoëToft (2002) acerca do inglês falado no sul da Inglaterra, a forma com schwa é produzida, no máximo, em 6,42\% das ocorrências.

\footnotetext{
2 “Essas pronúncias são altamente artificiais e nunca seriam ouvidas em uma fala casual de conversação".
} 
Levando em consideração esses dados, é compreensível assumirmos, como faz um número de fonólogos (JONES, 1962; GIGERISH, 1992; JUCOVY, 2003), que a lateral ocupa o núcleo da sílaba já no input, e que o schwa do output seja produto de epêntese. A escrita de palavras como <apple>, <bottle> e <Bible> também parece fornecer evidência para isso, uma vez que $0<\mathrm{l}>$ nesses casos segue imediatamente um grafema consonantal e o grafema <e> está no final da sílaba (posição na qual geralmente não possui qualquer representação sonora). Entretanto, tomar essa posição seria ignorar dois fatos importantes não mencionados até então. O primeiro é que as formas com schwa são relativamente frequentes na fala de crianças (WELLS, 1995, apud ODA, 2014). Isto sugere que o schwa seja suprimido no processo de aquisição e não inserido. Isso é reforçado pelo segundo fato, o de que as formas silábicas da lateral são foneticamente mais longas que as laterais que ocupam a coda silábica, sugerindo assim a ocorrência de peso compensatório após a perda de um elemento pertencente ao input.

Além disso, não podemos ignorar a similaridade entre a distribuição da lateral silábica e a do schwa: ambos ocorrem apenas em sílabas não tônicas. E o pequeno grau de variação existente no inglês L1 é entre a silábica e o schwa. Quando a lateral está acompanhada de vogais cheias ou acentuadas, ela sempre emerge na coda. É importante notar também que nesta posição, ao contrário de palavras com lateral silábica, existe um alto grau de variação entre [ł], [l], e [w] em vários dialetos do inglês. Portanto, há uma relação especial entre o schwa e a lateral silábica na língua inglesa. ${ }^{3}$ Por fim, em oposição à escrita das palavras usadas como exemplo no parágrafo anterior, existe também um grande número de palavras nas quais a lateral silábica ocorre e há uma vogal escrita antes de <l>; alguns exemplos são <national $>$, < angel $>$, e < original $>$.

A questão da produção das crianças ainda pode ser questionada, uma vez que, em referência aos dados, podemos dizer que esta ocorrência é produto de epêntese como estratégia para evitar estruturas marcadas nos estágios iniciais do processo de aquisição. Entretanto, como explicar a similaridade na distribuição e o peso compensatório? A posição de que as consoantes silábicas ocupam a forma subjacente não oferece qualquer tipo de resposta teórica aos dados empíricos, enquanto que a forma com schwa possui evidências fortes em seu favor. Com base nestas observações,

\footnotetext{
${ }^{3}$ Não somente no inglês, entretanto. 0 mesmo tipo de distribuição acontece no alemão, por exemplo.
} 
nos sentimos compelidos a adotar nesse estudo a forma /əl/ como sendo o input das sílabas que emergem foneticamente com $[\nmid]$ no inglês.

\subsection{A lateral no português brasileiro}

Para os falantes do português brasileiro (doravante PB), a lateral em posição de núcleo, assim como qualquer outra consoante nessa posição, é um fenômeno inexistente. Apenas vogais ocupam o núcleo de sílabas em PB. A lateral fica, portanto, restrita às posições de ataque e coda; além disso, ela só emerge foneticamente na primeira dessas posições silábicas. Quando em coda, a lateral sofre categoricamente um processo de vocalização ${ }^{4}$, dando lugar à semivogal $[\mathrm{w}]$. Assim, palavras como /pa'pel/ são realizadas foneticamente como [pa'pew].

\subsection{A aquisição da lateral silábica por falantes de PB}

Os estudiosos de aquisição de L2 concordam que a gramática da interlíngua de um aprendiz de LE em seu estado inicial seja igual à gramática de sua L1. Sendo assim, ao considerarmos as descrições realizadas acima sobre o comportamento da consoante lateral em PB (língua de partida), podemos esperar que, ao produzir um output para um input do inglês cuja forma seja /pəl/, o falante de PB inicialmente produzirá [pəw], uma vez que, na gramática do PB, uma vogal deve sempre ocupar o núcleo silábico e a lateral é vocalizada quando em posição de coda.

Se considerarmos apenas o fenômeno de vocalização e esquecermos a forte restrição que falantes de PB possuem em relação a consoantes em núcleo silábico, estaremos erroneamente igualando teoricamente o processo de aquisição da lateral silábica do inglês à aquisição da lateral que deve ocorrer em coda como nas palavras /tכ:l/ 'tall', ou /gəul/ 'goal' as quais vimos acima. Isso pode nos levar a problemas metodológicos na hora de estudar a realização da lateral do inglês por falantes de PB. Ao se preocupar unicamente com o fenômeno de vocalização ao estudar palavras como <castle> e <channel>, Hahn (2010, p. 86) é surpreendida com o seguinte resultado:

\footnotetext{
${ }^{4}$ Com exceção da fala de algumas comunidades no sul do Brasil as quais preservam as laterais na coda, tanto alveolares quanto velares. (HAHN, 2010, p. 27)
} 
Nem todas as ocorrências de /l/ em núcleo foram realizadas pelos informantes como tal. Em muitos casos, se não na maioria, os informantes produziram um segmento vocálico na posição de núcleo ([ə], por exemplo) e /l/ em coda. Não realizamos um controle sistemático sobre essas ocorrências, pois nossa intenção era manter a classificação destes dados como "C_." e assim o fizemos.

Todavia, embora a autora tenha desconsiderado uma forte restrição de PB que certamente afetaria a produção na interlíngua dos informantes (resultado do peso secundário recebido pela lateral silábica), o trabalho pioneiro de Hahn (2010) nos oferece dados antes inexistentes e que são relevantes por duas razões. A partir dos resultados encontrados, podemos perceber que a natureza do processo de aquisição das formas-alvos com laterais silábicas é diferente daquelas com a lateral na coda. A grande diferença entre os dois processos é que antes de pensar na vocalização ou não da lateral na própria posição de núcleo, ou até mesmo sobre qual alofone da lateral irá emergir, devemos primeiramente nos perguntar se a lateral irá de fato ocupar o núcleo silábico na interlíngua. Do ponto de vista teórico-metodológico, as possibilidades de variação não se restringem a $[\mathrm{w}],[\nmid]$, ou [l] (como é o caso para lateral-coda), mas a [w], [ł], [l], [əw], [əł⿱一兀), ou [əl]. Apesar de os dados de Hahn apontarem para [əł⿱ ] como a variante mais recorrente na interlíngua dos brasileiros, é plausível que, uma vez em coda, a lateral possa ser vocalizada como acontece em PB. A autora não controlou ou codificou esses dados.

Apesar do alto grau de variabilidade que pode teoricamente surgir a partir de um mesmo input (/əl/) fazer parecer caótico e inviável um estudo que controle metodologicamente todas essas variantes, a situação não é tão paralisante. Se olharmos com atenção para as variantes, logo percebemos que [əw], [əł⿱一⿱㇒⿵冂⿰丨丨寸], ou [əl] não são formas silábicas, e que a alternância entre essas formas é uma consequência de a lateral estar na coda. Se pensarmos a partir de uma perspectiva teórica derivacional, por exemplo, poderíamos dizer que, por o schwa não sofrer uma regra de elisão e assim a lateral permanecer na coda, o elemento fica sujeito às regras que se aplicam a ele naquela posição, e daí as três variantes.

Isto posto, acreditamos que a questão primordial no estudo de aquisição da lateral silábica por aprendizes brasileiros de inglês é a posição na sílaba em que a lateral irá ocorrer foneticamente; afinal, isto é o que faz da lateral ser silábica ou não. A forma articulatória na qual a lateral é realizada é, portanto, uma questão secundária. 
Entretanto, essa divisão é feita para tornar viável o controle metodológico da pesquisa, pois toda variação deve ser em última instância descrita e explicada. A consequência de todas estas colocações é que, em nossa pesquisa empírica (seção 3), dividimos a codificação e quantificação dos dados em duas etapas. Na primeira, controlamos a variação binária [l] x [əl], na qual [l] significa qualquer alofone da lateral (ou a semivogal) contanto que seja silábica ([w], [ł] ou [l]), e [əl] qualquer vogal no núcleo com qualquer alofone da lateral na coda ([əw], [əł]], ou [əl]). Em outras palavras, primeiramente nos preocupamos apenas em verificar se houve uma vogal no núcleo ou não. Na segunda etapa de codificação, selecionamos apenas as ocorrências com vogal no núcleo e verificamos se ocorreu vocalização ou não quando a lateral permaneceu em coda. A não codificação da qualidade clara ou escura da lateral será explicada em nossos resultados e a questão será considerada em nossa análise via SOT.

\section{Metodologia}

Nesta seção, apresentaremos a metodologia do estudo que conduzimos acerca da realização da lateral silábica do inglês por aprendizes brasileiros de língua inglesa como LE. Os resultados deste estudo servirão tanto como base para nosso modelo gramatical, como também para testá-lo empiricamente posteriormente (como veremos na análise via SOT na seção 3). Entretanto, a proposta não se limita ao mesmo.

A pesquisa foi desenvolvida a partir do modelo metodológico advindo dos estudos variacionistas labovianos (LABOV, 1972), tal como sugerido por Bailey (2005) para os estudos de Aquisição de Segunda Língua. As etapas procedimentais foram as seguintes: 1 - seleção de variáveis; 2 - seleção dos informantes; 3 - preparação dos instrumentos de coleta, codificação; 4 - rodagem estatística dos dados. Após a obtenção dos primeiros resultados, recodificamos parte dos dados de acordo com uma nova variável dependente. Vejamos o que foi feito em cada uma das etapas.

\subsection{Seleção das variáveis}

Nossa variável dependente foi a lateral silábica como forma-alvo e, como mencionado no último parágrafo da seção 2 , nossas variantes binárias foram na verdade 
dois grupos de variantes: as que apresentavam a lateral silábica e as que não apresentavam (com uma vogal em seu núcleo).

Quanto a variáveis linguísticas, selecionamos contexto fonológico anterior (em relação ao ponto de articulação), contexto fonológico anterior (em relação à maneira de articulação), contexto fonológico posterior (em classes maiores: consoante, vogal ou pausa), e posição ortográfica de $<\mathrm{l}>$ (antes de uma vogal, como em <people $>$, ou depois, como em <national>). Do lado das variáveis extralinguísticas, escolhemos controlar o informante, o nível de proficiência (três níveis), e conhecimento explícito de fonologia (sim ou não). Todas as variáveis extralinguísticas (com exceção de informante) são de caráter híbrido, pois não constituem de fato uma categoria social; entretanto, elas estão de certa maneira relacionadas ao nível de escolaridade e ao tipo de escolaridade dos informantes, uma vez que estamos lidando com um contexto em que o inglês está sendo aprendido como LE.

Não iremos aqui detalhar e discutir o porquê da escolha de cada uma das variáveis e qual a relevância teórica que elas possuem dentro do modelo variacionista de análise linguística. ${ }^{5}$ A relevância dessa pesquisa para a proposta do presente artigo está nos dados e nos padrões variáveis revelados a partir da metodologia variacionista. Nosso trabalho aqui será informar e testar nosso modelo formal a partir destes dados. Isto será feito na seção 4.

\subsection{Seleção dos informantes}

O corpus da pesquisa foi construído a partir da produção de doze informantes. Cada informante foi alocado em um dentre três diferentes níveis de proficiência: básico, intermediário e avançado. Dentro de cada grupo, cada qual com quatro informantes, houve uma subdivisão entre aprendizes com conhecimento explícito sobre a estrutura silábica do inglês e aprendizes sem esse tipo de conhecimento, totalizando dois membros dentro de cada subgrupo. Podemos visualizar a estratificação desses informantes na tabela a seguir:

\footnotetext{
${ }^{5}$ Para isso, ver ALVES \& LUCENA (no prelo).
} 
Tabela 1 - Estratificação dos informantes

\begin{tabular}{|c|c|c|c|c|c|c|c|c|c|c|c|c|}
\hline I & 01 & 02 & 03 & 04 & 05 & 06 & 07 & 08 & 09 & 10 & 11 & 12 \\
\hline $\mathbf{P}$ & \multicolumn{4}{|c|}{ Básico } & \multicolumn{4}{|c|}{ ntermediário } & \multicolumn{4}{|c|}{ Avançado } \\
\hline C.E. & S & $\mathrm{S}$ & $\mathrm{N}$ & $\mathrm{N}$ & $S$ & $S$ & $\mathrm{~N}$ & $\mathrm{~N}$ & $S$ & $\mathrm{~S}$ & $\mathrm{~N}$ & $\mathrm{~N}$ \\
\hline
\end{tabular}

$\mathrm{I}$ = informante; $\mathrm{P}=$ proficiência; $\mathrm{C} . \mathrm{E}$. = conhecimento explícito $(\mathrm{S}=\operatorname{sim}, \mathrm{N}=$ não).

Para delimitar o nível de proficiência, utilizamos o Oxford Placement Test (ALLAN, 2004), um teste de proficiência validado em mais de quarenta países. Para obter o grupo que havia sido exposto à instrução explícita em fonologia, selecionamos seis informantes (dois de cada nível de proficiência) que haviam cursado a disciplina Fonética e Fonologia da Língua Inglesa I pelo curso de graduação em Letras - Inglês da Universidade Federal da Paraíba. A estrutura silábica do inglês é um dos conteúdos constituintes do curso acima mencionado, e todas as consoantes silábicas do inglês são apresentadas aos alunos de forma teórica e prática. Tal conhecimento é também abordado em um dos testes da disciplina.

\subsection{Instrumentos e coleta de dados}

Para obter as produções de palavras que possuem a lateral silábica na língua alvo, fizemos uso de um instrumento de leitura que consistia de dois textos impressos. Para gravação da leitura dos textos, utilizamos um gravador Olympus WS-300. Os informantes puderam ler o texto antes de gravar.

\subsection{Codificação e rodagem estatística dos dados}

A análise do corpus foi realizada de maneira perceptual. Após a codificação de todas as ocorrências, utilizamos o programa Goldvarb X (SANKOFF; TAGLIAMONTE; SMITH, 2005) para rodagem estatística dos dados. A análise acústica pelo programa PRAAT (BOERSMA \& WEENINK, 2012) está em progresso. ${ }^{6}$ Após a obtenção dos resultados, passamos a reanalisar somente as ocorrências em que uma vogal ocupou o núcleo silábico formando uma nova variável dependente: o /l/ em coda. Observamos, portanto, a vocalização ou não do elemento, como discutido no final da seção 1.

\footnotetext{
${ }^{6} \mathrm{~A}$ análise acústica vem ajudando principalmente a termos uma maior visibilidade acerca da ressonância das laterais a partir de F2.
} 


\section{Resultados e discussões}

Nesta seção, apresentaremos os dados empíricos obtidos a partir da pesquisa descrita em Metodologia (2). Na tabela abaixo, podemos observar a frequência global obtida na primeira rodagem de dados referente às formas com consoantes silábicas versus vogais no núcleo:

Tabela 2 - Frequência global da primeira rodada de dados ${ }^{7}$

\begin{tabular}{|c|c|c|}
\hline Variante & Aplicação/Total & Porcentagem \\
\hline Vogal no núcleo & $220 / 331$ & $66.5 \%$ \\
\hline Consoante no núcleo & $111 / 331$ & $33.5 \%$ \\
\hline
\end{tabular}

O resultado geral corrobora os dados de Hahn (2010), pois o núcleo silábico é ocupado por uma vogal (quase sempre o schwa) na maioria das produções da interlíngua dos aprendizes brasileiros. Entretanto, isto não se aplica a todos os níveis de proficiência. Nossos dados apresentam um padrão parecido (o valor de aplicação/default se refere à realização de uma vogal no núcleo):

Tabela 3 - Distribuição dos dados da primeira rodagem por nível de proficiência

\begin{tabular}{|c|c|cc|}
\hline Variantes & Aplicação/Total & Porcentagem & Peso Relativo \\
\hline Básico & $89 / 108$ & $82.4 \%$ & 0.68 \\
\hline Intermediário & $94 / 111$ & $84.7 \%$ & 0.72 \\
\hline \hline Avançado & $37 / 112$ & $33.0 \%$ & 0.15 \\
\hline
\end{tabular}

O programa computacional de base variacionista nos revela pesos relativos significativos em relação à variável nível de proficiência. Os aprendizes de nível básico e intermediário tendem a produzir formas com vogais no núcleo (com pesos relativos de 0.68 e 0.72 respectivamente), enquanto que a forma com a lateral silábica é a mais frequente entre os aprendizes de nível avançado (peso relativo de 0.15 para a produção com vogal no núcleo). Em outras palavras, até o nível intermediário, a interlíngua do aprendiz parece estar em conformidade com a gramática do $\mathrm{PB}$, e somente no nível avançado os aprendizes parecem adquirir a forma alvo e se aproximar mais da gramática do inglês.

\footnotetext{
7 Tab. 2 e Tab. 3 foram reproduzidas do trabalho de ALVES \& LUCENA (no prelo).
} 
Devido ao alto número de ocorrência de vogais em núcleo nos aprendizes de nível básico e intermediário e como descrito na seção de metodologia (2), fizemos uma segunda codificação com apenas aquelas ocorrências (220 tokens). Como esperávamos produções distintas entre os níveis básico e intermediário, e, como só controlamos na primeira codificação a presença ou não de uma vogal no núcleo ${ }^{8}$, passamos a acreditar que, embora o núcleo silábico fosse ocupado na maioria da produção dos dois grupos pelo schwa, a diferença talvez estivesse na coda. Nossa segunda hipótese previa que os informantes de nível básico produziriam mais [əw] e os de nível intermediário [əł⿱一⿻上丨]] (evitando a vocalização). Porém, fomos novamente surpreendidos com os seguintes resultados ${ }^{9}$ :

Tabela 4 - Frequência global da segunda rodada

\begin{tabular}{|c|c|c|}
\hline Variante & Aplicação/Total & Porcentagem \\
\hline əw & $188 / 211$ & $89.1 \%$ \\
\hline əł & $23 / 211$ & $10.9 \%$ \\
\hline
\end{tabular}

Vemos acima que [əw] é o grande favorito dos aprendizes brasileiros de inglês, até mesmo entre os avançados (aplicação se refere a [əw], e a não aplicação a [əł⿱一⿱㇒⿵冂⿰丨丨寸]):

Tabela 5 - Distribuição dos dados da primeira rodada por nível de proficiência

\begin{tabular}{|c|c|c|c|}
\hline Variantes & Aplicação/Total & Porcentagem & Peso relativo \\
\hline Básico & $75 / 82$ & $91.5 \%$ & 0.53 \\
\hline Intermediário & $86 / 93$ & $92.5 \%$ & 0.57 \\
\hline Avançado & $27 / 36$ & $75.0 \%$ & 0.24 \\
\hline
\end{tabular}

No nível básico, encontramos apenas 7 produções de [əł⿱ ], 7 no intermediário, 9 no avançado. Confirma-se, portanto, uma enorme semelhança na produção dos dois níveis mais baixos de proficiência.

Existe uma última observação que deve ser feita em relação aos nossos dados para que possamos ter uma visão geral da grande variabilidade presente na interlíngua dos brasileiros em relação à aquisição da lateral silábica. Embora não tenhamos feito

\footnotetext{
${ }^{8}$ Esperávamos que os aprendizes intermediários se aproximassem mais da gramática do inglês. Entretanto, eles apresentaram à primeira vista basicamente o mesmo tipo de produção dos aprendizes de nível básico.

${ }^{9}$ Tínhamos 220 ocorrências com vogal no núcleo, como visto nas tabelas anteriores. Porém, 9 delas foram produzidas como [pli] (em palavras como 'simple') e por isso retiradas da rodagem estatística pois não apresentam coda.
} 
uma terceira codificação e a rodada dos dados, notamos ainda que, dentre as poucas ocorrências de produções sem uma vogal no núcleo (17 ocorrências), os aprendizes de nível intermediário preferiram [pw] (na última sílaba de palavras como 'simple' ou 'apple') em detrimento de [pł] majoritariamente. A produção da lateral com uma ressonância predominantemente clara foi insignificante em termos numéricos, tanto no núcleo quanto na coda (nem chegou a ocorrer na coda).

Por fim, podemos resumir as realizações dos aprendizes de diferentes níveis encontradas em nossa pesquisa da seguinte maneira:

Tabela 6 - Distribuição geral da ordem de frequência de todas as variantes por nível de proficiência

\begin{tabular}{|c|c|c|c|c|c|c|c|c|}
\hline Variantes & $1^{\circ}$ & $2^{\circ}$ & $3^{\circ}$ & $4^{\circ}$ & $X$ & $X$ & $X$ & $X$ \\
\hline Básico & [әw] & [w] & - & - & [əłt] & {$[\mathrm{t}]$} & [l] & [əl] \\
\hline Intermediário & [әш] & [w] & - & - & [əł] & [ł] & [1] & [əl] \\
\hline Avançado & [ł] & [w] & [әł] & [әw] & & & [1] & [əl] \\
\hline
\end{tabular}

Os números ordinais na primeira linha indicam a frequência das variantes na produção dos informantes e os respectivos níveis de proficiência dos aprendizes que as produziram. $\mathrm{O}$ ' $\mathrm{X}$ ' indica a irrelevância do número de ocorrências ou da não ocorrência da variante.

\section{Análise via SOT}

Até então apresentamos os dados que conhecemos sobre a distribuição de outputs no inglês L1 e da interlíngua de aprendizes brasileiros de inglês para um dado input /əl/. Nesta seção faremos uma análise dos dados via SOT de maneira a propor um modelo que descreva e explique os fenômenos descritos, respondendo às questões norteadoras levantadas na seção introdutória deste artigo.

Embora os fenômenos relacionados às consoantes silábicas do inglês ainda não tenham sido devidamente formalizados, uma importante generalização sobre essas consoantes já foi feita. A partir da escala de duas harmonias [[núcleo/vogal>núcleo/liquida>núcleo/nasal...]] (resultado do alinhamento harmônico 
entre a escala de proeminência da posição da sílaba e da escala de sonoridade), a OT naturalmente determina uma hierarquia fixa de restrições de marcação como [[...*Núcleo/Nasal>>*Núcleo/Líquida>>*Núcleo/Vogal] $]$.

Em relação às consequências empíricas dessa hierarquia fixa, McCARTHY (2002, p. 22) afirma "[...]the nucleus hierarchy accounts for the implicational universal that some languages have only vowel nuclei (Italian) and some have both liquid and vowel nuclei (English bottle), but no language has only liquid nuclei[...]".

Podemos, portanto, postular que no caso do inglês (e também do alemão, por exemplo), línguas nas quais as consoantes silábicas são produzidas apenas no final de palavras e em sílabas em que o schwa está presente no input, é a relação de dominação

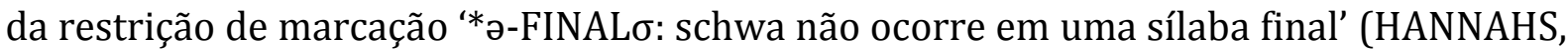
2007, p. 32) e a hierarquia fixa acima que licenciam certas classes de consoantes da língua (e não outras) a ocuparem a posição de núcleo. Além disso, é a relação da restrição com a escala que torna a previsão empírica dessa correta. No inglês, consoantes nasais também podem ocupar o núcleo silábico, logo as líquidas também podem. Porém, fricativas e oclusivas não são permitidas no núcleo. Propomos então o seguinte ranqueamento para o inglês: [[*Núcleo/Fricativa $>{ }^{*}{ }^{*}$ -

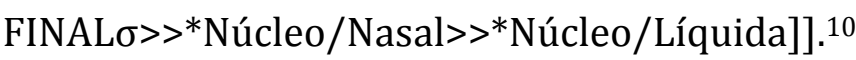

Entretanto, em se tratando da lateral, não podemos assegurar que [1] resulte da avaliação do ranqueamento e apenas essas duas restrições, ou seja, [[*ə-

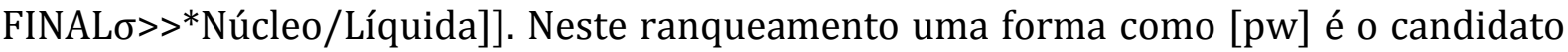
ótimo ${ }^{11}$ :

\footnotetext{
${ }^{10}$ É interessante observar que no estudo de TOFT (2002), o autor descobriu que as consoantes nasais ocorrem menos no núcleo que a lateral. Isto encontra uma explicação natural na SOT uma vez que *Núcleo/Nasal se encontra mais próxima de ${ }^{*}$ ə-FINAL $\sigma$ que *Núcleo/Líquida, permitindo assim que *Núcleo/Nasal passe a dominar *ə-FINAL $\sigma$ com mais frequência do que *Núcleo/Líquida.

${ }^{11}$ Importante: em todos os tableaux deste trabalho, nosso input /pəl/ se refere, por exemplo, à última sílaba de palavras como 'people' ou 'apple'. Porém, a escolha de /p/ não importa. Nem importa se o elemento silabificado ficará de fato no onset das sílabas do output. Nossa intenção em colocar apenas uma sílaba nos tableaux foi de criar um foco nos elementos que estamos discutindo. Uma segunda consideração importante é que sempre que 'l' aparecer como candidato a output, sua ressonância (clara ou escura) não está sendo especificada ainda. Utilizamos o alofone do /l/ claro porque tínhamos que colocar algum $<\mathrm{l}>$. Explicaremos a questão da preferência dos informantes pela ressonância escura apenas no final desta seção, e ficará claro que até lá a discussão não era relevante para o resto da análise.
} 
Tableau 1

\begin{tabular}{|r||c|c|}
\hline$/ \mathrm{p} \partial \mathrm{l} /$ & $* \partial-\mathrm{FINAL \sigma}$ & $*$ NUCLIQUID \\
\hline \hline $\mathrm{pl}$ & & $* !$ \\
\hline $\mathrm{pw}$ & & \\
\hline
\end{tabular}

Precisamos ainda de uma restrição de fidelidade como '/l/ = [l]: /l/ corresponde ao output [1]' (BOROWSKY \& HOVARTH, apud JUCOVY, 2003, p. 10) para garantir a permanência da lateral no output:

Tableau 2

\begin{tabular}{|r||c|c|c|}
\hline$/ \mathrm{p} ə 1 /$ & $* \partial-F I N A L \sigma$ & $/ 1 /=[1]$ & $*$ NUCLIQUID \\
\hline \hline pl & & & $*$ \\
\cline { 3 - 4 } $\mathrm{pw}$ & & $* !$ & \\
\hline
\end{tabular}

Por fim, para dar conta da baixa, mas ainda existente variação entre [l] e [əl] na língua inglesa, precisamos de uma restrição que permita formas com schwa no output. *NucLiquid como C1 favoreceria formas como [əl] ou [əw] em detrimento de [l], mas não de $[w]$, uma vez que ela não viola *NucLiquid. Propomos, portanto, a inserção de $\mathrm{N}=\mathrm{V}$ no ranqueamento. Assim, [əl] resulta da seguinte gramática:

Tableau 3

\begin{tabular}{|c|c|c|c|c|}
\hline /pəl/ & $\mathrm{N}=\mathrm{V}$ & ə-FINAL $\sigma$ & $/ 1 /=[1]$ & *NUCLIQUID \\
\hline pl & $* !$ & & & $*$ \\
\hline pw & $*$ ! & & $*$ & \\
\hline$\sigma \mathrm{pal}$ & & * & & \\
\hline
\end{tabular}

Esse resultado só é obtido, em termos de SOT, nas raríssimas ocasiões em que no momento de avaliação o valor de ranqueamento de $\mathrm{N}=\mathrm{V}$ for maior que o de *ə-FINAL $\sigma$,

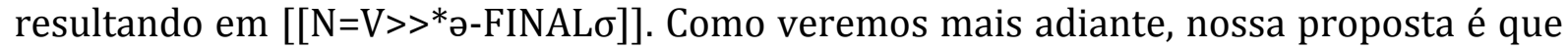
a possível interseção entre as duas distribuições normais (com valor de noise 5.0) só aconteça na distância de aproximadamente dois desvios padrões do valor de 
ranqueamento de ambas as restrições. Portanto, em quase todas as avaliações do input /əl/, obtém-se o seguinte resultado para a gramática do inglês:

Tableau 4

\begin{tabular}{|r||c|c|c|c|}
\hline$/ \mathrm{p} ə \mathrm{l} /$ & ə-FINAL $\sigma$ & $\mathrm{N}=\mathrm{V}$ & $/ 1 /=[1]$ & $*$ NUCLIQUID \\
\hline \hline $\mathrm{pw}$ & & $*$ & & $*$ \\
\hline $\mathrm{p}$ & & $*$ & $* !$ & \\
\hline & $* !$ & & & \\
\hline
\end{tabular}

Nota-se também que, para manter [1] como forma quase categórica, a distância dos valores de ranqueamento de /l/=[l] e *NucLiquid também deve ser considerável, evitando assim [[*NucLiquid $>* / 1 /=[1]]]$; que resultaria na seleção de $[\mathrm{pw}]$ como candidato mais harmônico. Muito embora isso venha acontecendo em alguns dialetos nativos do inglês L1, como vimos no estudo de Jucovy (2003) acerca do inglês australiano, tal fenômeno fortalece nosso argumento, uma vez que encontra uma simples e intuitiva explicação no mesmo. Isto posto, a partir de agora consideraremos que essa deve ser a gramática alvo do aprendiz brasileiro de inglês. Respondemos assim nossa primeira questão norteadora.

Começaremos agora a considerar qual o ranqueamento responsável pelas formas observadas em nossos dados dos aprendizes brasileiros; afinal, a forma mais frequente encontrada foi [əw] e ainda não a consideramos. Para tanto, precisamos primeiramente entender por que a escolha de [w] no núcleo vem acontecendo para podermos pensar em sua aparição na coda, afinal o glide é uma dentre infinitas alternativas que GEN pode gerar para ocupar o lugar da lateral.

Para [w] ser o candidato ótimo (ocupando o núcleo silábico) em nosso ranqueamento de quatro restrições acima, precisaríamos da seguinte hierarquia desta

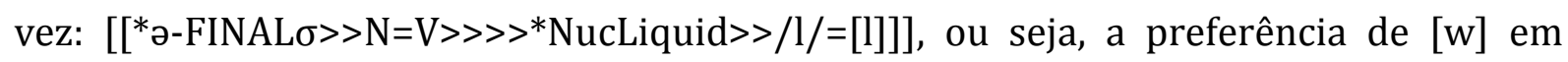
detrimento de [l] se dá por conta da disputa entre a restrição de marcação envolvendo as líquidas (que inclui a lateral) e a restrição de fidelidade também em relação à lateral. Portanto, [w] é o candidato ótimo, porque pode ser gerado por GEN, mas não há nada que force a escolha por um glide. [w] é menos harmônico nesse caso que [I], ou qualquer outra vogal, pois essa forma não viola C1 nem C2 enquanto [w] viola C2. Para explicar a 
escolha de $[w]$ em detrimento de qualquer outra vogal/glide, precisamos ampliar nosso ranqueamento com 1) a restrição de marcação '*nV[-stress]: toda vogal com valor de sonoridade mais alto que o das vogais médias centrais não são permitidas no núcleo de sílabas não acentuadas' (KHANJIAN, 2009, p. 6) e 2) a restrição de fidelidade de traço IDENT (dorsal) (KAGER, 1999). Segue a avaliação do novo ranqueamento:

Tableau 5

\begin{tabular}{|c|c|c|c|c|c|c|}
\hline /pəl/ & IDENT(DORSAL) & $* \mathrm{nV}[$-stress $]$ & $* \partial$-FINAL $\sigma$ & $\mathrm{N}=\mathrm{V}$ & *NUCLIQUID & $/ 1 /=[1]$ \\
\hline $\mathrm{pl}$ & & & & $*$ & $* !$ & \\
\hline pol & & & $* !$ & & & \\
\hline$\Leftrightarrow \mathrm{pw}$ & & & & $*$ & & $*$ \\
\hline pI & $* !$ & $*$ & & & & $*$ \\
\hline pD & & $* !$ & & & & $*$ \\
\hline pj & $* !$ & & & $*$ & & $*$ \\
\hline
\end{tabular}

A posição fortemente dominante da restrição de fidelidade ao traço dorsal elimina qualquer possibilidade de [j] emergir no output. A restrição de marcação, por outro lado, é responsável por banir qualquer vogal que não seja [ə] do núcleo silábico (com exceção de vogais que não possuem o traço dorsal, pois essas provocam uma violação crítica antes mesmo de ser avaliada por C2, como é o caso com [I]). Explicamos assim a preferência por [w] em núcleo em um ranqueamento completo. Entretanto, não há ainda lugar para a forma [əw]. Com as restrições que trabalhamos até então, em todas as possibilidades em que [ə] está presente no candidato mais harmônico, a lateral deve necessariamente ser o acompanhante daquele candidato:

Tableaux 5 e 6

\begin{tabular}{|c|c|c|c|c|}
\hline /pel/ & $\mathrm{N}=\mathrm{V}$ & *ə-FINAL $\sigma$ & *NUCLIQUID & $/ 1 /=[1]$ \\
\hline $\bar{\sigma}=\mathrm{pel}$ & & $*$ & & \\
\hline pəw & & $*$ & & *! \\
\hline pə & & $*$ & & $* !$ \\
\hline
\end{tabular}

\begin{tabular}{|c|c|c|c|c|}
\hline /pel/ & $\mathrm{N}=\mathrm{V}$ & $*$ *-FINAL $\sigma$ & $/ 1 /=[1]$ & *NUCLIQUID \\
\hline$C=$ pel & & * & & \\
\hline pəw & & $*$ & $* !$ & \\
\hline pə & & $*$ & $* !$ & \\
\hline
\end{tabular}


Ao analisarmos os dois tableaux acima, logo percebemos que a restrição decisiva é $/ \mathrm{l} /=[1]$. Assim sendo, propomos a inserção da restrição 'Co=GL/V (BOROWSKY \& HOVARTH, apud JUCOVY, 2003, p. 10): a coda da sílaba mais harmônica é um glide ou uma vogal' em nosso ranqueamento. Essa restrição foi uma das utilizadas pelos autores acima para explicar a vocalização da lateral na coda e no núcleo que ocorre no inglês australiano. Desconsideramos até então a análise feita pelos autores acerca da vocalização da lateral em palavras como 'bottle', pois eles adotaram a visão de que a lateral silábica já está presente no input (/bbtl/ para a palavra mencionada), visão essa da qual discordamos veementemente devido às razões apontadas na seção 1.1 deste artigo. Além disso, o objeto de estudo foi apenas a vocalização, e não a emergência de silábicas.

Com essa restrição no ranqueamento, o output mais frequente entre os brasileiros (principalmente nos níveis básico e intermediário) pode se tornar o candidato ótimo no momento da seleção, como vemos no tableau abaixo:

Tableau 7

\begin{tabular}{|c|c|c|c|c|c|}
\hline /pəl / & $\mathrm{N}=\mathrm{V}$ & ə-FINAL $\sigma$ & *NUCLIQUID & $\mathrm{CO}=\mathrm{GL} / \mathrm{V}$ & $/ 1 /=[1]$ \\
\hline $\mathrm{pl}$ & $* !$ & & $*$ & $*$ & \\
\hline pəw & & $*$ & & & $*$ \\
\hline pw & $* !$ & & & $*$ & $*$ \\
\hline pəl & & $*$ & & $* !$ & \\
\hline
\end{tabular}

Podemos afirmar, portanto, que as restrições propostas até então e os diferentes ranqueamentos entre as mesmas são capazes de explicar a existência de todos os outputs que encontramos nos dados empíricos tanto na produção de L1 como de LE (no caso de brasileiros). Nosso trabalho agora será descrever e explicar, a partir do Gradual Learning Algorithm, o caminho que a gramática da interlíngua do aprendiz brasileiro de inglês percorre em direção à aquisição da forma alvo. Teremos sempre em mente a partir de agora os dados de nossa pesquisa, muito embora também façamos referência aos de Hahn (2010) e outros (JUCOVY, 2003; TOFT, 2003).

Os aprendizes brasileiros de nível básico de proficiência de nossa pesquisa produziram [əw] em $89.1 \%$ das ocorrências. Isso confirmou nossas expectativas 
teóricas, pois a gramática do aprendiz ainda é quase a mesma ou a mesma de sua L1. No $\mathrm{PB}$, como vimos antes, a forma [əw] é de se esperar, uma vez que a língua não admite consoantes no núcleo e a lateral é categoricamente vocalizada na posição pós-nuclear. Sendo assim, assumimos que a gramática apresentada no tableau 6 acima é a de PB, configurando o estágio inicial de aquisição de [l] através do input /əl/. Outro fator importante que deve informar nosso ranqueamento estocástico é que a única forma em variação com [əw] nesse nível de proficiência é [w], enquanto outras formas como [əł]] e [ł] aparecem em um número insignificante de vezes (quando aparecem). Isso significa que, embora a gramática do aprendiz de nível básico já consiga excluir uma vogal de baixa sonoridade como [ə] do núcleo mesmo em poucas ocorrências, a vocalização do elemento 3 do input ainda é praticamente categórica.

Com isso em mente, atribuímos valores de ranqueamento mais próximos entre as

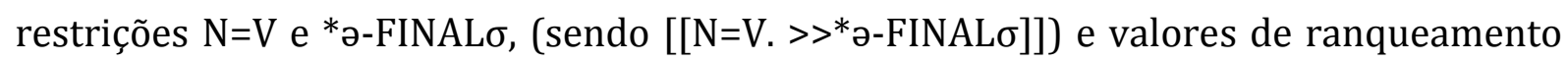
mais distantes entre *NucLiquid e $/ \mathrm{l} /=[\mathrm{l}]$ (sendo[[*NucLiquid $>>/ \mathrm{l} /=[1]]]$ ) (até mesmo porque $\mathrm{Co}=\mathrm{GL} / \mathrm{V}$ está ranqueado entre estas duas últimas). Asseguramos assim que, mesmo quando *ə-FINAL $\sigma$ dominar $\mathrm{N}=\mathrm{V}$ em algumas avaliações, *NucLiquid ainda estará dominando /l/=[1], não permitindo que [l] resulte do trabalho de EVAL e sim [w] (que é a segunda variante mais encontrada na fala dos aprendizes básicos). Por fim, não podemos esquecer que a frequência de [w] não deve ser alta, portanto, embora $\mu \mathrm{C} 1(\mathrm{~N}=\mathrm{V})^{12}-\mu \mathrm{C} 2\left({ }^{*}\right.$ - - FINAL $\left.\sigma\right)<\mu \mathrm{C} 3\left({ }^{*}\right.$ NucLiquid) $-\mu \mathrm{C} 5(/ \mathrm{l} /=[1])$, a diferença entre $\mu \mathrm{C} 1 \mathrm{e}$ $\mu \mathrm{C} 2$ deve ser ainda grande o suficiente para não permitir que C2 domine C1 com frequência no momento de avaliação. C5 dominará C3 ainda menos, por isso a quase inexistente ocorrência de [əl] ou [1]. Os valores aleatórios de ranqueamento $(\mu)$ escolhidos foram $[[\mathrm{N}=\mathrm{V} \quad(100)>>*$ ə-FINAL $\sigma \quad$ (94) $>>*$ NucLiquid(80)>>Co$\mathrm{V} / \mathrm{GL}(72)>>/ \mathrm{l} /=[\mathrm{l}]$ (65)]] com noise 5.0. O importante na proposta é o valor das diferenças entre os valores de ranqueamento das restrições, assim, independentemente dos números escolhidos (que, como dito antes, são aleatórios), deve-se preservar as seguintes equações: $\mu \mathrm{C} 1-\mu \mathrm{C} 2=6, \mu \mathrm{C} 2-\mu \mathrm{C} 3=14, \mu \mathrm{C} 3-\mu \mathrm{C} 4=8, \mu \mathrm{C} 4-\mu \mathrm{C} 5=7$.

Observa-se que, como proposto acima, a diferença entre $\mu \mathrm{C} 1$ e $\mu \mathrm{C} 2$ é menor que a diferença entre $\mu \mathrm{C} 3$ e $\mu \mathrm{C} 5$, mas a diferença entre $\mu \mathrm{C} 1$ e $\mu \mathrm{C} 2$, que é de 6 pontos, sendo,

\footnotetext{
${ }^{12} \mu=$ mean de uma distribuição normal, equivalente ao valor de ranqueamento de uma restrição em SOT.
} 
portanto, maior que o valor de noise (5) (valor que é equivalente à área do primeiro desvio padrão (68\%) no mecanismo de distribuição normal do PRAAT). 0 resultado dos outputs para 101 trials (selecionamos o mesmo número de trials que o número de ocorrências que coletamos de aprendizes de nível básico) do ranqueamento que acabamos de definir foi o seguinte:

Tabela 7 - Comparação na distribuição dos dados

\begin{tabular}{|c|c|}
\hline Resultados da computação & Resultados reais \\
\hline \begin{tabular}{r|c} 
pw & 28 \\
pal & 3 \\
pəw & 70
\end{tabular} & \begin{tabular}{r|l} 
pw & $\cong 19$ \\
pəl & 7 \\
pəw & 75 \\
pl & $\cong 1$
\end{tabular} \\
\hline
\end{tabular}

Comparando os dois resultados, percebemos que a computação do ranqueamento estocástico proposto faz com que a máquina obtenha resultados similares aos dos informantes.

Os dados dos aprendizes de nível intermediário (como vimos no início desta seção) são altamente semelhantes aos do básico, portanto, aprendizes intermediários parecem não apresentar qualquer mudança na gramática que acabamos de propor. Poderíamos conceber tal semelhança como indício de fossilização, mas os dados dos aprendizes avançados parecem mostrar que, em relação a esse fenômeno, embora seja necessário muito input, os aprendizes brasileiros de inglês como LE apresentam mudanças na gramática de suas interlínguas em estágios mais avançados de proficiência. A grande questão aqui, em se tratando de um contexto no qual o inglês está sendo aprendido como LE, é que não apenas formas da L1 serão tomadas como evidência positiva para o algoritmo com o qual estamos trabalhando. Assim, voltaremos à questão mais tarde. Por hora, passaremos a analisar as mudanças ocorridas na gramática para explicar as produções dos aprendizes de nível avançado.

Ao contrário dos níveis mais baixos, as formas sem vogal no output são mais frequentes, e a preferência pela manutenção da lateral no output é maior que a produção do glide. Destarte, encontramos, nesse estágio, [l] como a forma mais comum, 
seguida de perto por $[\mathrm{w}]^{13}$. Isto significa dizer que a gramática dos aprendizes avançados está mais próxima da do inglês L1 (pois a lateral silábica já é a forma mais recorrente); porém, ela apresenta uma variação diferente da do inglês e uma frequência menor da lateral silábica. Como então explicar a diferença entre a gramática dos aprendizes básicos/intermediários de nosso estudo e a dos avançados? Como uma se transforma na outra?

Acreditamos que o Gradual Learning Algorithm (ou GLA) oferece uma resposta efetiva para essa questão. Ao acessar o input /əl/ através do output [l] (1 ${ }^{\circ}$ passo do GLA), o algoritmo gera uma avaliação para o input ( $2^{\circ}$ passo do GLA). Assim, se o ranqueamento no momento de avaliação for [[N=V >>*ə-FINAL $\sigma>>^{*}$ NucLiquid $>>$ Co$\mathrm{V} / \mathrm{GL}>>/ \mathrm{l} /=[\mathrm{l}]]$ ] (o ranqueamento mais comum entre os aprendizes de níveis básico e intermediário), o algoritmo identificará uma incompatibilidade (durante a etapa de comparação $-3^{\circ}$ passo do GLA) entre a forma produzida pelo ranqueamento atual e o dado a que foi exposto:

Tableau 8

\begin{tabular}{|c|c|c|c|c|c|}
\hline /pel / & $\mathrm{N}=\mathrm{V}$ & $*$ *FINAL $\sigma$ & *NUCLIQUID & $\mathrm{CO}=\mathrm{GL} / \mathrm{V}$ & $/ 1 /=[1]$ \\
\hline$\dot{ } \overrightarrow{p l}$ & $* !$ & & * & * & \\
\hline$G=$ pəw & & * & & & * \\
\hline
\end{tabular}

Como podemos ver, para chegar à gramática que produz [l], ou seja, [[*əFINAL $\sigma>>\mathrm{N}=\mathrm{V}>>/ \mathrm{l} /=[\mathrm{l}]>>*$ NucLiquid $>>\mathrm{Co}=\mathrm{GL} / \mathrm{V}]]$, uma série de ajustes deve proceder ( $4^{\circ}$ passo do GLA):

Tableau 9

\begin{tabular}{|c|c|c|c|c|c|}
\hline /pel / & $\mathrm{N}=\mathrm{V}$ & *ə-FINAL $\sigma$ & *NUCLIQUID & $\mathrm{CO}=\mathrm{GL} / \mathrm{V}$ & $V / 1 /=[1]$ \\
\hline $\mathrm{pl}$ & $* !$ & $\rightarrow$ & $* \longrightarrow$ & $* \rightarrow$ & \\
\hline$=$ pow & & $\leftarrow *$ & & & 梅* \\
\hline
\end{tabular}

É interessante observar que, seguindo o mecanismo do algoritmo, o valor de ranqueamento de todos os candidatos será modificado quando a forma produzida for

\footnotetext{
${ }^{13}$ Como dito anteriormente, não fizemos uma terceira rodada apenas com as formas sem vogais no núcleo. Porém, anotações realizadas durante a etapa de codificação para a primeira rodada registraram um grande número de ocorrências de [w] no núcleo.
} 
[əw], pois nenhuma marca de violação teve de ser cancelada no ajuste. Isto demonstra o poder do argumento desse ranqueamento.

À medida que os ajustes que resultam dessa comparação acontecem gradualmente, é esperado que *ə-FINAL $\sigma$ adquira um valor de ranqueamento maior que o de $\mathrm{N}=\mathrm{V}$ antes que /l/=[l] obtenha um valor maior que *NucLiquid. Isso acontece porque, como vimos anteriormente, a diferença entre os valores de $\mu$ das restrições $N=V$

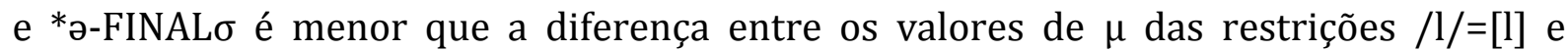
*NucLiquid. Dessa maneira, haverá um estágio na gramática produzida pelo algoritmo em que *ə-FINAL $\sigma$ dominará $\mathrm{N}=\mathrm{V}$ sem que $/ \mathrm{l} /=[1]$ já domine *NucLiquid. 0 resultado é o ranqueamento no qual [pw] será o candidato ótimo mais frequente:

Tableau 10

\begin{tabular}{|r||c|c|c|c|c|}
\hline$/$ pəl $/$ & e-FINAL $\sigma$ & $\mathrm{N}=\mathrm{V}$ & $*$ NuCLIQUID & $/ 1 /=[1]$ & $\mathrm{CO}=\mathrm{GL} / \mathrm{V}$ \\
\hline \hline $\mathrm{pl}$ & & $*$ & $* !$ & & $*$ \\
\hline Tow & & $*$ & & $*$ & $*$ \\
\hline $\mathrm{pw}$ & $* !$ & & $*$ & $*$ & \\
\hline $\mathrm{p} 2 \mathrm{l}$ & $* !$ & & & & $*$ \\
\hline
\end{tabular}

Essa gramática explicaria a produção do nosso informante de número 11, que parece produzir mais [w] no núcleo que [1]. Os outros três informantes do mesmo grupo de proficiência apresentam uma distribuição inversa a essa, produzindo mais [l] que [w]. Essa distribuição dos dados da maioria dos informantes do grupo avançado também encontra uma explicação no processo de aprendizagem contínua do algoritmo, pois quando [w] se torna a variante mais frequente (como no tableau 10 acima) e continua sendo comparado com o dado do ambiente [l] (input/əl/), em algum momento $\mu$ de $/ \mathrm{l} /=[1]$ será maior que $\mu$ de *NucLiquid (tornando [l] o candidato ótimo mais frequente):

Tableau 11

\begin{tabular}{|c|c|c|c|c|c|}
\hline /pel/ & - FINAL $\sigma$ & $\mathrm{N}=\mathrm{V}$ & *NUCLIQUID & $/ 1 /=[1]$ & $\mathrm{CO}=\mathrm{GL} / \mathrm{V}$ \\
\hline$\sqrt{\mathrm{pl}}$ & & $\not$ & $* ! \longrightarrow$ & & $*$ \\
\hline$G=\mathrm{pw}$ & & $*$ & $\leftarrow$ & * & $*$ \\
\hline
\end{tabular}


Tableau 12

\begin{tabular}{|r||c|c|c|c|}
\hline$/ \mathrm{p} \partial \mathrm{l} /$ & $\partial-F I N A L \sigma$ & $\mathrm{N}=\mathrm{V}$ & $/ 1 /=[1]$ & $*$ NUCLIQUID \\
\hline \hline $\mathrm{p} 1$ & & $*$ & & $*$ \\
\hline $\mathrm{pw}$ & & $*$ & $* !$ & \\
\hline
\end{tabular}

Destacamos, entretanto, que nesse estágio que corresponde às produções da maioria dos aprendizes do grupo de proficiência avançado e ilustrado no tableau 12 , o valor de ranqueamento de $/ l /=[1]$ já é maior que o de *NucLiquid mas ainda estão próximos o suficiente para produzir [w] com uma frequência considerável (assim como nos nossos resultados). Propomos, assim, uma distância de apenas 4 pontos (um valor menor que o noise 5.0) entre essas duas restrições.

Para concluir nossa resposta às questões norteadoras de número 2 e 3 , podemos afirmar que a maioria dos informantes de nível avançado está mais próxima da gramática de L1 que todos os outros, mas ainda não alcançaram o estado final do processo. Se continuarmos seguindo o algoritmo, sempre que [w] for selecionado como output ótimo, a avaliação fará com que o valor do ranqueamento de $/ 1 /=[1]$ continue crescendo e o de *NucLiquid diminuindo até chegar o ponto em que a escolha de $[w]$ como candidato ótimo quase nunca aconteça. Propomos a seguinte gramática estocástica para o inglês L1: [[N=V>>*ə-FINAL $\sigma>>1 /=[1]>>* N u c L i q u i d]]$ (tableau 4) com os seguintes valores $\mu \mathrm{C} 1-\mu \mathrm{C} 2=12,0 ; \mu \mathrm{C} 2-\mu \mathrm{C} 3=28 ; \mu \mathrm{C} 3-\mu \mathrm{C} 4=25$ (noise $=5.0$ ). Esta seria a gramática do inglês e o estágio final do processo de aprendizagem do algoritmo. Em simulação no Praat com 1000 outputs a partir dessa gramática obtivemos: [pl] 956 = 95\% - [pw] $2=0,2 \%$ - [pəl] $42=4,2 \%$. Estes dados se aproximam da média de porcentagem da produção de [pəł] e [pł] encontrada no estudo de Toft (2005) acerca do inglês falado no sul da Inglaterra. Nota-se novamente que a gramática é capaz de gerar [pw]; forma emergente no inglês australiano (JUCOVY, 2003).

Por fim, explicamos a preferência quase categórica por uma lateral mais escura em quase todos os outputs que analisamos, adicionando parte do argumento de ranqueamento de Hayes (1998, p. 19) aos ranqueamentos que propomos até então: 
Tableau 13

\begin{tabular}{|c|c|c|c|c|c|c|c|}
\hline /pəl/ & $\mathrm{N}=\mathrm{V}$ & ${ }^{*}$-FINAL $\sigma$ & $/ 1 /=[1]$ & *NuCLIQUID & $\mathrm{CO}=\mathrm{GL} / \mathrm{V}$ & [ł] IS / V & $/ 1 /$ is DARK! \\
\hline $\mathrm{pt}$ & $*$ & & & * & $*$ & & \\
\hline pl & $*$ & & & $*$ & $*$ & & $* !$ \\
\hline
\end{tabular}

/l/ is DARK faz com que a ressonância escura seja a favorita na disputa entre [l] e [ł], e a restrição [ł] IS/V_ também garante esta ressonância seja avaliada como mais harmônica quando a disputa é entre [pəl] e[pəł].

\section{Considerações preliminares}

A principal contribuição deste trabalho está na sua dimensão epistemológica. Mesmo admitindo a necessidade na literatura em fonologia de mais dados empíricos com frequências precisas acerca do fenômeno da lateral silábica, salientamos que a gramática por nós proposta é capaz (como vimos) de descrever e explicar a existência de qualquer um dos outputs (para o mesmo input /əl/) já observados em dados reais de fala: [əw], [w], [əł], [ł], [əl], [l]. Destarte, mesmo que futuras análises acústicas de nossos dados ou de novos dados revelem frequências diferentes para as variantes, tudo que precisaremos fazer será repensar os valores de ranqueamento das restrições, mas não mudá-las.

Os argumentos de ranqueamento com base nas restrições que utilizamos (as quais já existiam na literatura em OT) foram capazes de demonstrar não somente uma relação natural entre as variantes do inglês L1 ou da interlíngua de brasileiros, como também, através do componente estocástico da gramática e dos mecanismos do GLA, encontra uma elegante explicação para diferentes sotaques do inglês L1 e para as mudanças na gramática da interlíngua dos aprendizes. Essa explicação de diversos fenômenos a partir de um mesmo mecanismo gramatical reforça o caráter universalista da Teoria da Otimalidade e consequentemente fornece evidências para a universalidade da gramática enquanto sistema cognitivo.

Para concluir, apontamos para problemáticas futuras que surgem a partir de reflexões acerca dos resultados do presente estudo. Primeiramente, embora já tenhamos brevemente proposto uma explicação nos moldes da OT (partindo da escala de 
McCarthy (2002) - seção 4 deste trabalho) para generalizações em relação às consoantes silábicas nas línguas do mundo, não podemos perder de vista que no inglês, por exemplo, a frequência da ocorrência de $\left[\mathrm{r}_{1}\right]$ ou [m] $]$ são menores que as da lateral silábica (ROACH, 2000). Além disso, até dentro de uma mesma classe fonológica, como a das nasais, [ṇ] é muito menos frequente que [m] e [ñ ] (TOFT, 2003). Dessa forma, essas assimetrias no comportamento das consoantes silábicas tanto entre diferentes classes como dentro de uma mesma classe fonológica devem ser investigadas e elucidadas.

Em segundo lugar, se acreditarmos na força dedutiva do GLA em relação à cognição linguística humana, acabamos por admitir que o papel dos inputs que um aprendiz de segunda língua recebe e classifica como evidência positiva da língua-alvo é de grande importância para entendermos melhor o fenômeno de fossilização na aquisição (como parece ter acontecido com nossos informantes de nível intermediário, que apresentaram quase as mesmas produções que os de nível básico). Se entendermos as mudanças gramaticais da interlíngua como resultados de um ajuste gradual no valor de restrições universais, e que esse reajuste é feito a partir de uma comparação com a forma classificada pelo aprendiz como evidência positiva, passamos a entender o curso de desenvolvimento da interlíngua (no nível fonológico pelo menos) como dependente em grande parte do input ao qual o aprendiz é exposto, o qual considera evidência positiva. Daí talvez se desfaça a necessidade que diversos linguistas têm em traçar uma forte distinção entre a natureza do processo de aquisição de língua estrangeira (muitas vezes chamado de 'aprendizagem') e de segunda língua. A principal diferença talvez seja apenas o input tomado como evidência positiva pelos aprendizes. Isto posto, o papel do input se configura como um tema pertinente para estudos futuros.

\section{Referências}

ALLAN, D. Oxford Placement Test 1. Oxford University Press, 2004.

ALVES, F. C.; LUCENA, R. M. Análise Variacionista do /l/ em Posição de Núcleo por Aprendizes de Inglês como L2. Anais - VIII Congresso Internacional da ABRALIN. No prelo.

BAYLEY, R. Second Language Acquisition and Sociolinguistic Variation. Intercultural Communication Studies XIV - 2, 2005. 
BOERSMA, P. Optimality-T theoretic learning in the Praat program. IFA proceedings 23: 17-35. 1999.

BOERSMA, P.; HAYES, B. Empirical tests of the Gradual Learning Algorithm. Linguistic Inquiry 32, p. 45-86, 2001.

BOERSMA, P.; WEENIK, D. Praat: doing phonetics by computer - version 5.3.32. 2012.

GIGERICH, H. J. English Phonology: An Introduction. Cambridge: Cambridge University Press, 1992.

HAHN, Laura Helena. A realização da lateral /l/ no inglês por falantes do português brasileiro. 2010. 102 f. Dissertação (Mestrado em Letras) - Universidade Federal do Rio Grande do Sul, Porto Alegre, 2010.

HANNAHS, S. J. Welsh vowel mutation: an optimality analysis. Newcastle upon Tyne: School of English Literature, Language and Linguistics, Newcastle University. Newcastle Working Papers in Linguistics, 12 \& 13. 2007.

HAYES, B. Gradient well-formedness in Optimality Theory. Department of Linguistics, University of California, Los Angeles. 1998.

JONES, D. Outline of English Phonetics. 9. ed. Cambridge W. HEFFER \& SONS LTD, Great Britain, 1962.

JUCOVY, K. An Optimality-Theory-Based Analysis of Variable/l/-Vocalization in Australian English. 2003. Disponível em: http://hdl.handle.net/10066/10304. Acesso em: 30 jun. 2014

KAGER, R. Optimality Theory. Cambridge: Cambridge University Press, 1999.

KHANJIAN, H. Stress Dependent Vowel Reduction. Proceedings of Berkeley Linguistics. Society. 2009.

LABOV, W. Sociolinguistic Patterns. Pennsylvania University Press. 1972.

McCARTHY, J. A Thematic Guide to Optimality Theory. Cambridge: Cambridge University Press. Pp. xiv+317. 2002.

ODA, H. Further Evidence on the Underlying Schwa of Syllabic Consonants in Present-Day English: Against the Form without Schwa. Disponível em: <http://www14.ocn.ne.jp/ toda/proceeding jel syllcons.pdf>. Acesso em: 30 jun. 2014.

PIÑEROS, C. Syllabic-Consonant Formation in Traditional New Mexico Spanish. Disponível em: $<$ http://roa.rutgers.edu/files/635-0104/635-PINEROS-0-0.PDF $>$. Acesso em: 30 jun. 2014 
PRINCE, A.; SMOLENSKY, P. Optimality Theory: Constraint interaction in generative grammar. Technical Report, Rutgers University and University of Colorado at Boulder, 1993. Revised version published by Blackwell, 2004.

ROACH, P. English Phonetics and Phonology. 3. ed. Cambridge: Cambridge University Press, 2000.

SANKOFF, D.; TAGLIAMONTE, S.; SMITH, E. Goldvarb $X$ : a variable rule application for Macintosh and Windows. Department of Linguistics, University of Toronto, 2005.

TOFT, Z. The Phonetics and Phonology of some Syllabic Consonants in Southern British English. School of Oriental and African Studies University of London, 2002.

Recebido em junho de 2014.

Aceito em outubro de 2014. 